\title{
Facial symmetry and the perception of beauty
}

\author{
GILLIAN RHODES \\ University of Western Australia, Nedlands, Perth, Western Australia \\ and University of Canterbury, Christchurch, New Zealand \\ and \\ FIONA PROFFITT, JONATHON M. GRADY, and ALEX SUMICH \\ University of Canterbury, Christchurch, New Zealand
}

\begin{abstract}
Evolutionary, as well as cultural, pressures may contribute to our perceptions of facial attractiveness. Biologists predict that facial symmetry should be attractive, because it may signal mate quality. We tested the prediction that facial symmetry is attractive by manipulating the symmetry of individual faces and observing the effect on attractiveness, and by examining whether natural variations in symmetry (between faces) correlated with perceived attractiveness. Attractiveness increased when we increased symmetry, and decreased when we reduced symmetry, in individual faces (Experiment 1), and natural variations in symmetry correlated significantly with attractiveness (Experiments 1 and $1 \mathrm{~A}$ ). Perfectly symmetric versions, made by blending the normal and mirror images of each face, were preferred to less symmetric versions of the same faces (even when those versions were also blends) (Experiments 1 and 2). Similar results were found when subjects judged the faces on appeal as a potential life partner, suggesting that facial symmetry may affect human mate choice. We conclude that facial symmetry is attractive and discuss the possibility that this preference for symmetry may be biologically based.
\end{abstract}

The question of what makes a face attractive, and whether our preferences come from culture or biology, has fascinated scholars for centuries. Variation in the ideals of beauty across societies and historical periods suggests that standards of beauty are set by cultural convention. Recent evidence challenges this view, however, with infants as young as 2 months of age preferring to look at faces that adults find attractive (Langlois et al., 1987), and people from different cultures showing considerable agreement about which faces are attractive (Cunningham, Roberts, Wu, Barbee, \& Druen, 1995; Jones \& Hill, 1993; see Langlois \& Roggman, 1990, for a review).

These findings raise the possibility that some standards of beauty may be set by nature rather than culture. Consistent with this view, specific preferences have been identified that appear to be part of our biological rather than our social heritage (Langlois \& Roggman, 1990; Langlois, Roggman, \& Musselman, 1994; Perrett, May, \& Yoshikawa, 1994; Rhodes \& Tremewan, 1996). For example, average facial configurations are attractive (Langlois \&

This research was supported by grants from the Department of Psychology, University of Canterbury, the Australian Research Council, and the University of Western Australia. We thank Graham Byatt, Ian McLean, Johanna Roberts, and Leslie Zebrowitz for stimulating discussions about this work, and Rotem Kowner, Nicola Bruno, Randy Larsen, Leslie Zebrowitz, and an anonymous reviewer for helpful comments on an earlier version of the manuscript. We also thank Graham Byatt for assistance with stimulus construction, Linda Jeffery for assistance with the figures, and Alison Clark and Catherine Hickford for assistance with data collection and statistical analysis in Experiment 1A. Correspondence should be addressed to G. Rhodes, Department of Psychology, University of Western Australia. Nedlands. Perth. WA 6907, Australia (e-mail: gill@psy.uwa.edu.au)
Roggman, 1990; Langlois et al., 1994; Rhodes \& Tremewan, 1996). ${ }^{1}$ Such a preference would be adaptive if stabilizing selection operates on facial traits (Symons, 1979), or if averageness is associated with resistance to pathogens, as some have suggested (Gangestad \& Buss, 1993; Thornhill \& Gangestad, 1993). ${ }^{2}$

Evolutionary biologists have proposed that a preference for symmetry would also be adaptive because symmetry is a signal of health and genetic quality (Palmer \& Strobeck, 1986; Parsons, 1990; Thornhill \& Møller, 1997; Watson \& Thornhill, 1994). Only high-quality individuals can maintain symmetric development in the face of environmental and genetic stresses. Symmetric bodies are certainly attractive to humans and many other animals (Brooks \& Pomiankowski, 1994; Concar, 1995; Møller \& Pomiankowski, 1993; Thornhill \& Gangestad, 1994; Watson \& Thornhill, 1994), but what about symmetric faces?

Biologists suggest that facial symmetry should be attractive because it may signal mate quality (Ridley, 1992; Swaddle \& Cuthill, 1995; Watson \& Thornhill, 1994). High levels of facial asymmetry in individuals with chromosomal abnormalities (e.g., Down's syndrome and Trisomy 14; for a review, see Thornhill \& Møller, 1997) are consistent with this view, as is recent evidence that facial symmetry levels correlate with emotional and psychological health (Shackelford \& Larsen, 1997). In this paper, we investigate whether people can detect subtle differences in facial symmetry and whether these differences are associated with differences in perceived attractiveness.

Recently, Kowner (1996) has reported that faces with normal levels of asymmetry are more attractive than perfectly symmetric versions of the same faces. ${ }^{3}$ Similar results have been reported by Langlois et al. (1994) and Sam- 
uels, Butterworth, Roberts, Graupner, and Hole (1994). Together, these studies suggest that symmetry is not attractive in faces. Other results, however, suggest that facial symmetry is attractive. In particular, natural variations in symmetry appear to covary with attractiveness (Grammer \& Thornhill, 1994; Jones \& Hill, 1993, for some ethnic groups; Zebrowitz, Voinescu, \& Collins, 1996; but see Jones \& Hill, 1993, for other ethnic groups; Langlois et al., 1994).

How can these two conflicting sets of results be reconciled? One possibility is that symmetry is attractive within the normal range of variation, but that perfect symmetry is not preferred. To understand why this might be so we need to distinguish between two kinds of asymmetry in faces: fluctuating and directional asymmetries. Fluctuating asymmetries are randomly distributed (with respect to the direction of the asymmetry) across individuals in a population, so that there is no consistent leftright bias in the population as whole. These asymmetries result from environmental or genetic stresses, and so may be reliable indicators of mate quality. Unlike fluctuating asymmetries, directional asymmetries have a consistent bias (to the left or right) across a population, are not produced by stresses during development, and are not potential indicators of mate quality. Directional asymmetries in faces include systematic biases for the left side of face to be larger (Previc, 1991) and more expressive (Borod, 1993) than the right. Because of these directional asymmetries, a face will be somewhat asymmetric in the absence of any stresses during development; that is, perfect symmetry will not be the norm. Therefore, it is possible that symmetry is attractive within the naturally occurring range of symmetry levels because variations within that range primarily reflect fluctuating asymmetries, but that perfect symmetry is unattractive because it is abnormal.

There is, however, another possible resolution of the two sets of results. The studies reporting that perfect symmetry is unattractive have compared normal faces with perfectly symmetric chimeras, constructed by reflecting each half of the face about the vertical midline (yielding two chimeras for each face). However, these chimeras may not be appropriate for assessing the attractiveness of perfect symmetry in faces because they can contain structural abnormalities that make them look strange. Part of the problem stems from the directional size asymmetry in faces, which means that for many faces, one chimera will be wider, and the other narrower, than a normal face (i.e., the chimeras may have widthheight aspect ratios outside the normal range). Features in the center of the face may also be abnormal in chimeras. For example, if the nose bends to one side of the face, then one chimera will have an abnormally wide and the other an abnormally narrow nose. An asymmetrically positioned mouth will generate similar distortions in the chimeras. Slight deviations from frontal views in the original photographs will compound these problems and introduce new abnormalities (e.g., the eyes may be- come abnormally narrow-set or widely spaced in the chimeras if there is any rotation around the main axis of the head). Given that attractiveness decreases with distortion from a prototypical facial configuration (see, e.g., Rhodes $\&$ Tremewan, 1996), these abnormalities are likely to make the chimeras unattractive, thus offsetting any preference for symmetry.

An alternative way to construct perfectly symmetric faces that does not introduce structural abnormalities is to blend the normal and mirror images of each face. This technique results in more natural looking symmetric faces that might well be considered attractive. We therefore decided to reassess the attractiveness of perfect symmetry using this technique to make perfectly symmetric faces. We also investigated whether the attractiveness of individual faces could be manipulated by varying their level of symmetry across a wider range of symmetry levels than is normally found in faces. This manipulation provides a strong test of how the level of symmetry influences facial attractiveness. It also tests Kowner's (1996) claim that observers are not tuned to perceive the low degree of asymmetry present in normal faces. If she is correct, observers should be unable to distinguish normal (asymmetric) faces from perfectly symmetric versions of the same faces.

Langlois and her colleagues (1994) used a similar technique to create symmetric versions of individual faces when they blended the forward and mirror images of 16 slightly different shots of each face to make a symmetric 32-image version of each face. However, they did not use these images to assess the attractiveness of symmetry. Rather, they compared these same-face composites with a composite made from 32 different individuals in a successful attempt to show that the attractiveness of composite or average faces was not simply the result of their symmetry or an artifact of combining multiple images.

\section{EXPERIMENT 1}

In Experiment 1, we varied the level of symmetry in individual faces and examined the effect on attractiveness. There were four versions of each face (Figure 1). One of these was a perfectly symmetric version created by blending the normal and mirror images of the face. The others were (1) a high-symmetry version, created by reducing the differences between the original face and its perfectly symmetric version by $50 \%,(2)$ the original face showing a normal level of symmetry, and (3) a lowsymmetry version, created by increasing the differences between the original face and its perfectly symmetric version by $50 \%$ (see below for details).

Subjects rated all versions of all the faces on attractiveness. If a preference for symmetry is part of our evolutionary heritage, as conjectured, then a preference for symmetry should ultimately influence mate choice. Therefore, we also asked subjects to rate opposite-sex faces on appeal as a potential life partner. The order of attractiveness and mate-choice ratings was counterbalanced across subjects. Finally, we asked subjects to rate all versions 

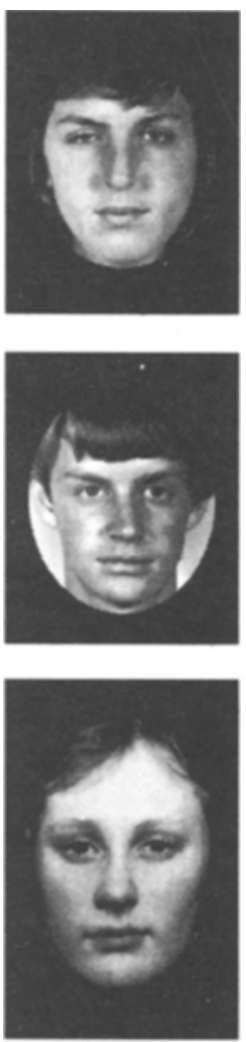

Low
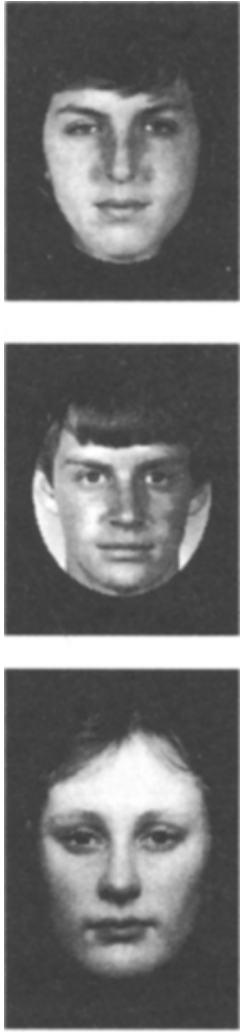

Normal
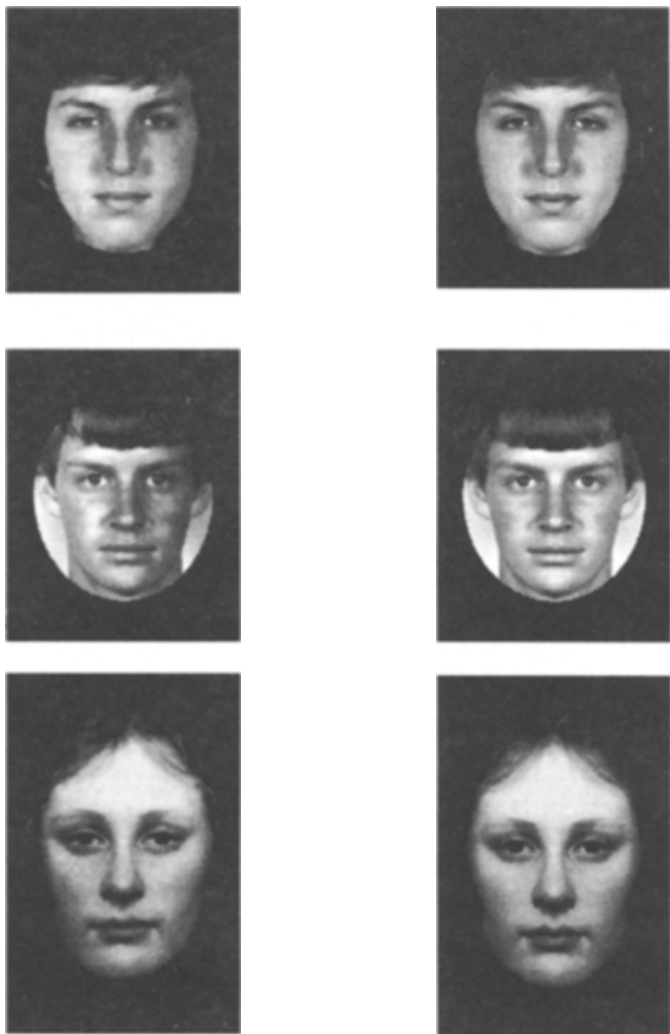

High
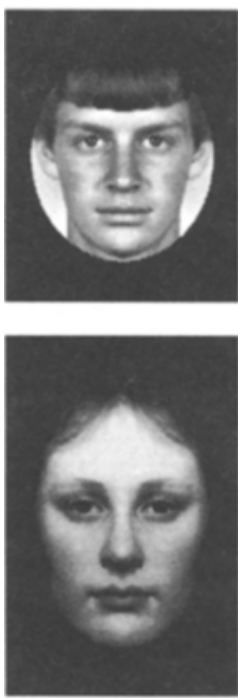

Perfect

\section{SYMMETRY}

Figure 1. Low-, normal-, high-, and perfect-symmetry versions for three faces from Experiment 1.

of all the faces on symmetry level to determine whether or not they were sensitive to different levels of symmetry in faces.

\section{Method}

Subjects. Sixty-four university students ( 32 males, 32 females) received $\$ 20$ each for participating.

Stimuli. Digitized black-and-white photographs of 48 young adult faces ( 24 males, 24 females) were used. All were frontal views, with neutral expressions, taken with symmetric lighting. Blemishes, earrings, clothing, and stray pieces of hair were removed using the cloning stamp tool in Photoshop. To correct for any slight deviations from verticality in the images, the best-fitting line (found by eye) through the midpoints of the lines joining the inner eye corners, the outermost edges of nose, and the outer corners of mouth was aligned to vertical. The midpoint between the eyes was centered on the screen before a face was manipulated. A fixed set of 120 landmark points (a subset of the 169 points used in Rhodes \& Tremewan, 1996) outlining the shape and position of the internal features and face outline was located on each face.

Perfectly symmetric versions were created using Gryphon's Morph software to average the normal and mirror images of each face. This procedure first averages the locations of the points and then averages the gray-level values in corresponding regions of the face (for details. see Beale \& Keil. 1995). To make high-and low-symmetry versions of a face, corresponding points are found (by the program) on the original face and its perfectly symmetric version, and all distances between the corresponding points are reduced (to create a high-symmetry version) or increased (to make a low-symmetry version) by $50 \%$. The gray levels from the original face are then mapped onto the new configuration. An oval mask hid most of the neck and the top of the head (Figure 1). The resulting face images measured approximately $8 \times 12 \mathrm{~cm}$ and had a resolution of 106 pixels per inch. One version of each face was assigned to each of four booklets, with booklets balanced for sex and symmetry level. Additional male-only and female-only booklets were created for use in the mate appeal ratings

Procedure. Each subject rated the attractiveness $(1=$ not at all attractive, $10=$ very attractive $)$ and symmetry $(1=$ not at all symmetric, $10=$ perfectly symmetric) of all four versions of all 48 faces (different versions in different booklets, as described above) and rated all four versions of all 24 opposite-sex faces for mate appeal ("How appealing is this person as a life partner?" $1=$ not at all appealing, $10=$ very appealing). Order of attractiveness and mate appeal ratings was counterbalanced with order of booklets. Symmetry ratings were always made last so that attention was not drawn to symmetry before the attractiveness and mate appeal ratings were made. Subjects were tested individually.

\section{Results and Discussion}

Reliability for attractiveness, mate appeal, and symmetry ratings was assessed separately for male and female subjects and faces. All ratings were highly reliable, with Cronbach alpha's ranging from .92 to .98 . Threeway analyses of variance (ANOVAs) were carried out on the mean attractiveness and symmetry ratings, with sex 
of subject as a between-subjects factor and sex of face and symmetry level as repeated measures factors. A twoway ANOVA was carried out on the mean mate appeal ratings, with sex of subject as a between-subjects factor and symmetry level as a repeated measures factor. For each analysis, planned $t$ tests were carried out to test for differences between symmetry levels.

Attractiveness ratings. There was a significant main effect of symmetry level $[F(3,186)=95.43, p<.0001]$, qualified by an interaction between symmetry level and sex of face $[F(3,186)=5.00, p<.003$; Figure 2, top]. Inspection of Figure 2 shows that despite the interaction be-
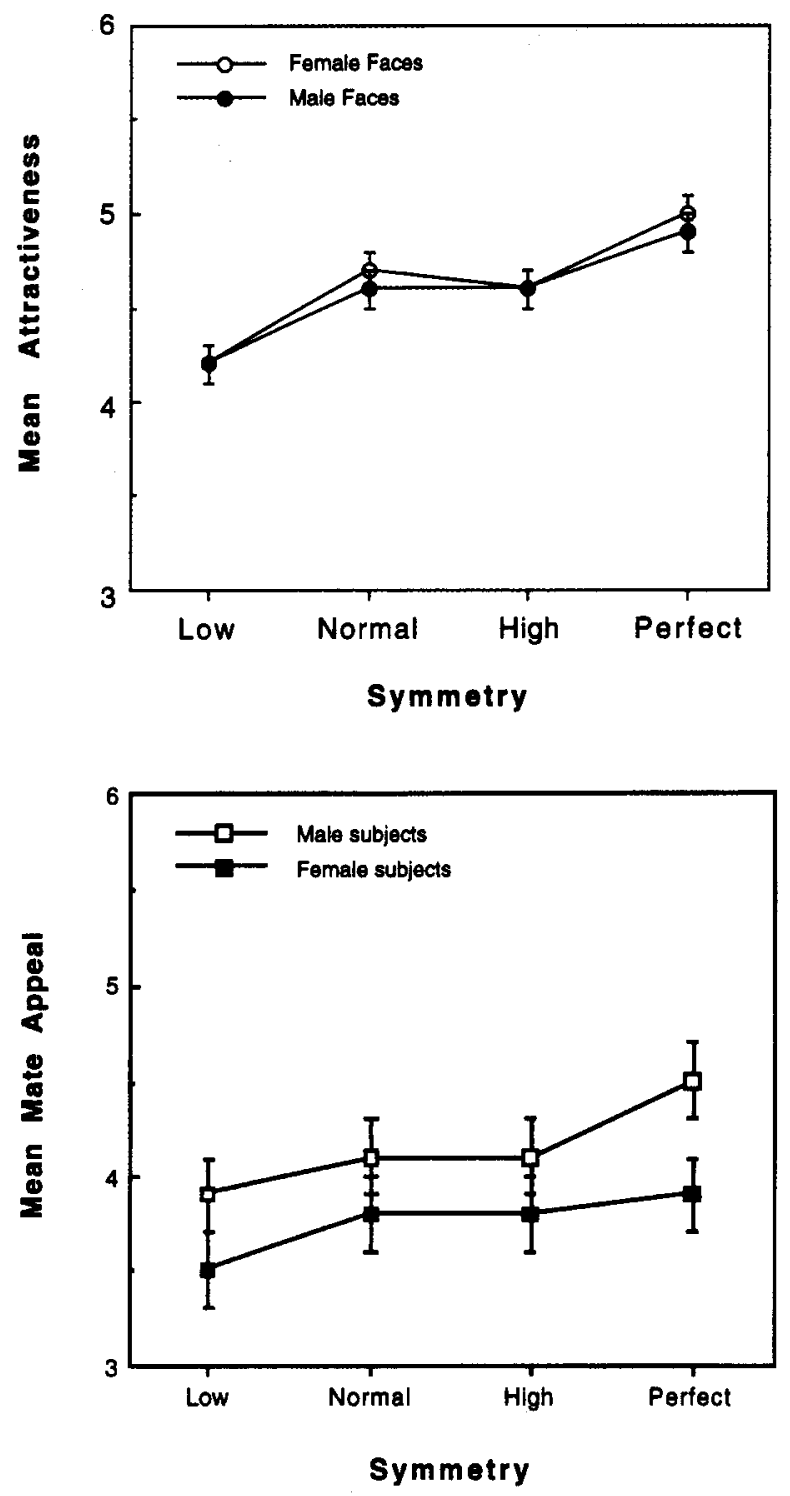

Figure 2. Mean attractiveness ratings (top) as a function of symmetry level and sex of face in Experiment 1. Mean mate appeal ratings (bottom) as a function of symmetry level and sex of subject in Experiment 1. SE bars are shown. tween symmetry level and sex of face, symmetry had qualitatively similar effects on the attractiveness of male and female faces. Simple tests of main effects showed that the effect of symmetry was significant for both male $[F(3,186)=53.63, p<.0001]$ and female $[F(3,186)=$ $88.89, p<.0001]$ faces. Perfect symmetry was significantly more attractive, and low symmetry significantly less attractive, than normal symmetry in both cases (all $p s<$ .001 ), but ratings of normal and high symmetry did not differ significantly for either male or female faces. None of the sex differences at the different symmetry levels were significant by simple tests of main effects (all $F \mathrm{~s}<2.52$ ).

Mate appeal ratings (opposite-sex faces only). As for attractiveness, there was a significant main effect of symmetry level $[F(3,186)=59.74, p<.0001]$, which was qualified by an interaction with sex of subject $[F(3,186)=$ $6.41, p<.002$; Figure 2, bottom]. Simple tests of main effects showed significant effects of symmetry level for both male $[F(3,186)=45.87, p<.0001]$ and female $[F(3,186)=20.28, p<.0001]$ subjects, but males found perfectly symmetric faces more appealing as potential life partners than did females $[F(1,62)=4.10, p<.05]$. The appeal of perfect symmetry was stronger for males than females, but males and females did not differ on any of the other symmetry levels (all $F \mathrm{~s}<1.99$ ). ${ }^{4}$ This sex difference is consistent with evidence that physical appearance plays a larger role in the mate choices of males than of females (Buss, 1987; Buss \& Schmitt, 1993). We note, however, that parental investment theory (Trivers, 1972) predicts the opposite sex difference. On that account, females should be more attuned to potential signals of mate quality than males because they make a greater parental investment than males.

Symmetry ratings. There was a significant main effect of symmetry level $[F(3,186)=429.54, p<.0001]$. Therefore, subjects were sensitive to the differences in symmetry levels within faces produced by our distortions, even though these differences were quite subtle (due to the approximate bilateral symmetry of faces). Symmetry level interacted with sex of face $[F(3,186)=16.80, p<.0001]$. Simple tests of main effects showed that the effect of symmetry was significant for both male $[F(3,186)=$ $373.04, p<.0001]$ and female $[F(3,186)=429.87, p<$ $.0001]$ faces, with symmetry ratings increasing significantly from one symmetry level to the next in both cases (male $M \mathrm{~s}=3.8,5.3,6.0$, and 8.3 ; female $M \mathrm{~s}=4.1,5.3$, 5.8 , and 8.3 for low, normal, high, and perfect symmetry, respectively; all $p s<.01$ ). There was a marginal interaction between symmetry and sex of subject $[F(3,186)=$ $2.25, p<.09$ ], with a wider range of ratings for males (3.8-8.4) than for females (4.0-8.2).

Correlations. Mean ratings were calculated for each face for each rating scale. Table 1 shows Pearson productmoment correlations between mean ratings of symmetry and attractiveness, symmetry and mate appeal, and attractiveness and mate appeal for the normal (undistorted) versions of the faces as well as for all versions (low, nor- 
Table 1

Pearson Product-Moment Correlations Between Mean Ratings of Symmetry and Attractiveness, Symmetry and Mate Appeal, and Attractiveness and Mate Appeal in Experiments 1 and $1 \mathrm{~A}$

\begin{tabular}{|c|c|c|c|c|c|c|}
\hline & \multicolumn{3}{|c|}{ Experiment 1 Subjects } & \multicolumn{3}{|c|}{ Experiment 1A Subjects } \\
\hline & $\begin{array}{c}\text { All } \\
\text { (All Faces) }\end{array}$ & $\begin{array}{c}\text { Male } \\
\text { (Female Faces) }\end{array}$ & $\begin{array}{c}\text { Female } \\
\text { (Male Faces) }\end{array}$ & $\begin{array}{c}\text { All } \\
\text { (All Faces) }\end{array}$ & $\begin{array}{c}\text { Male } \\
\text { (Female Faces) }\end{array}$ & $\begin{array}{c}\text { Female } \\
\text { (Male Faces) }\end{array}$ \\
\hline $\begin{array}{l}\text { Normal faces } \\
\text { Symmetry and attractiveness } \\
\text { Symmetry and mate appeal } \\
\text { Attractiveness and mate appeal }\end{array}$ & $\begin{array}{c}d f=46 \\
.33 \dagger \\
.26^{*} \\
.96 \S\end{array}$ & $\begin{array}{c}d f=22 \\
.36^{*} \\
.35^{*} \\
.99 \S\end{array}$ & $\begin{array}{l}d f=22 \\
.29 \\
.21 \\
.93 \S\end{array}$ & $\begin{array}{c}d f=46 \\
.29 \dagger \\
.13 \\
.858\end{array}$ & $\begin{array}{c}d f=22 \\
.41 \dagger \\
.42 \dagger \\
.93 \S\end{array}$ & $\begin{array}{c}d f=22 \\
.11 \\
-.10 \\
.77 \S\end{array}$ \\
\hline $\begin{array}{l}\text { All versions } \\
\text { Symmetry and attractiveness } \\
\text { Symmetry and mate appeal } \\
\text { Attractiveness and mate appeal }\end{array}$ & $\begin{array}{l}d f=190 \\
.36 \S \\
.27 \S \\
.95 \S\end{array}$ & $\begin{array}{c}d f=94 \\
.37 \S \\
.32 \ddagger \\
.98 \S\end{array}$ & $\begin{array}{c}d f=94 \\
.27^{+} \\
.22^{\dagger} \\
.93 \S\end{array}$ & $\begin{array}{c}d f=190 \\
.38 \S \\
.24 \S \\
.88 \S\end{array}$ & $\begin{array}{l}d f=94 \\
.43 \S \\
.33 \S \\
.93 \S\end{array}$ & $\begin{aligned} d f= & 94 \\
& .30^{+} \\
& .15 \\
& .85 \S\end{aligned}$ \\
\hline
\end{tabular}

Note-Separate correlations are shown for normal (undistorted) faces and all versions (low, normal, high, and perfect symmetry) of faces. Only opposite-sex faces were rated for mate appeal. $\quad{ }^{*} p<.10 . \quad{ }^{+} p<.05 . \quad{ }^{\ddagger} p<.01 . \quad \$_{p}<.001$.

mal, high, and perfect symmetry versions). In addition to overall correlations for all subjects and faces, correlations are also shown for opposite-sex ratings, which are more directly relevant to assessing the role of symmetry in mate choice.

For all faces (male and female) and ratings by all the subjects, all the correlations were significant except for that between mate appeal and symmetry in the normal faces, which was only marginally significant. These results clearly show that facial symmetry is attractive, and they corroborate our evidence for the attractiveness of symmetry obtained by direct manipulation of facial symmetry. Ratings of attractiveness and mate appeal were highly correlated, suggesting that ratings of attractiveness may have been based primarily on an assessment of sexual attractiveness, rather than other aspects of attractiveness, such as cuteness.

For opposite-sex ratings when all versions of the faces were considered, symmetry was again positively correlated with attractiveness and mate appeal. When only normal faces were considered, the correlations were similar, but failed to reach significance with the smaller number of faces. The preference for symmetry appeared to be stronger for males rating female faces than for females rating male faces.

The effect of symmetry on attractiveness did not depend on a face's initial attractiveness. The increase in attractiveness from the normal to the perfectly symmetric version of each face was uncorrelated with initial attractiveness $(r=.02, d f=46, \mathrm{n}$.s.). The corresponding correlation for mate appeal was .01 (n.s.). The scatterplots showed no hint of curvilinear relations between initial attractiveness or mate appeal and the enhancing effect of increased symmetry. These results do not support Langlois et al.'s (1994) conjecture that only exceptionally unattractive faces are improved by an increase in symmetry. They found that only very unattractive faces increased in attractiveness when made perfectly symmetric, but this result may reflect the use of perfectly symmetric chimeras. A normal face would have to be exceptionally unattractive to be rated less attractive than such strange looking images.
Our results indicate that facial symmetry is attractive. They should not, however, be taken to mean that symmetry is the only determinant of facial attractiveness. If it were, then perfectly symmetric faces would all have been judged equally attractive, and they were not. Mean ratings (averaged across subjects) for the perfectly symmetric images ranged from 5.5 to 7.9 (means for the normal faces ranged from 2.8 to 8.0 ), and these judgments were highly consistent, with male and female subjects' ratings correlating .91 $(d f=46, p<.001)$. Nor do these ratings indicate that symmetry is strikingly beautiful. Nevertheless, they do show that people are sensitive to different levels of symmetry in faces, and they generally find symmetry attractive.

\section{EXPERIMENT 1A}

In Experiment 1, each subject rated the faces on attractiveness, mate appeal, and symmetry, raising the possibility that the correlations reported above were inflated by carryover effects from one rating scale to another. ${ }^{5}$ Given that subjects rated a large number of images on each scale (blocked by scale), subjects would be unlikely to remember exactly how they had rated any particular image on an earlier scale. Nevertheless, there may be a more general carryover effect from thinking about a face's attractiveness (or mate appeal) before rating its symmetry, which could inflate the correlations between symmetry and attractiveness (or mate appeal). In Experiment 1A, therefore, we tested additional subjects to determine whether significant correlations between symmetry and attractiveness, and symmetry and mate appeal, would still be found when different subjects rated the faces on each scale.

\section{Method}

Subjects. One hundred and twenty-eight university students ( 64 males, 64 females) each received ether $\$ 5$ or course credit for participating. Of these, 64 ( 32 males, 32 females) rated the faces on symmetry, 32 ( 16 males, 16 females) on attractiveness, and 32 ( 16 males. 16 females) on mate appeal. Fewer subjects rated attractiveness and mate appeal because their data could be combined with the data from subjects in Experiment 1 who had rated those scales first 
Procedure. The procedure was identical to that of Experiment $1 \mathrm{ex}$ cept that each subject rated the faces on only one rating scale.

\section{Results and Discussion}

All ratings were highly reliable, with Cronbach alphas ranging from 0.93 to 0.99 . Mean symmetry, attractiveness, and mate appeal ratings were calculated for each image using ratings from separate groups of subjects. The symmetry ratings were calculated using the data from the new group of subjects only because no subjects in Experiment 1 had rated symmetry first. Attractiveness and mate appeal ratings were calculated by combining the data from the new subjects with data from those subjects in Experiment 1 who had rated that scale first.

Table 1 shows that the correlations between symmetry and attractiveness and between symmetry and mate appeal were very similar to those obtained in Experiment 1 . Of these 12 correlations (see Table 1, rows 2, 3, 6, and 7), 6 increased from Experiment 1 to Experiment $1 \mathrm{~A}$, and 6 decreased, suggesting that the use of the same subjects to make all the ratings did not consistently inflate the correlations. Most importantly, we replicated the significant correlations between symmetry and attractiveness for normal (undistorted) versions of the faces and for all versions of the faces. We also replicated the correlation between symmetry and mate appeal for all versions of the faces, but not the marginal correlation for normal faces found in Experiment 1. The correlations between attractiveness and mate appeal were high, but consistently lower than in Experiment 1.

As in Experiment 1, males appeared to have a stronger preference for symmetry in female faces than females had for symmetry in male faces. Males showed significant correlations between symmetry and attractiveness, and symmetry and mate choice, when rating normal female faces (cf. marginal correlations in Experiment 1). For females, neither correlation was significant for normal faces in either experiment, and the correlation between symmetry and mate appeal, which was significant for ratings of all versions of the faces in Experiment 1, was not significant.

As in Experiment 1, we found no support for the notion that symmetry enhances only unattractive faces. The difference in attractiveness between normal and perfectly symmetric versions of faces was uncorrelated with initial attractiveness $(r=-.20, d f=46$, n.s. $)$, and the corresponding correlation for mate appeal was -.10 (n.s.). The scatterplots showed no sign of curvilinear relationships.

Taken together, the correlations obtained in Experiments 1 and $1 \mathrm{~A}$ suggest that higher levels of symmetry enhance the attractiveness and mate appeal of a face. These effects are seen most clearly when an extended range of symmetry levels is used (all versions), but are still apparent when only the normal faces are considered. The opposite-sex ratings indicate that males and females both find symmetry attractive in opposite sex faces, but that the preference may be stronger for males than females. Only males showed replicable correlations between symmetry and mate appeal.

\section{EXPERIMENT 2}

In Experiment 1, we created perfectly symmetric faces by blending each face with its mirror image. This technique has the advantage of creating natural-looking symmetric faces. However, blends themselves may be more attractive than normal faces, because they are more average (Langlois \& Roggman, 1990), because they have smoother skin texture (Benson \& Perrett, 1992), or both. Therefore, our perfectly symmetric versions of faces could have been attractive because they were blends rather than because they were symmetric.

Two points argue against the interpretation of our results as solely due to blending artifacts. First, our perfectly symmetric faces were created by blending two face images, and two-face blends were not more attractive than the original faces in Langlois and Roggman's (1990) study. Sixteen faces had to be entered into the composites before they were more attractive than the original faces. Second, blending artifacts cannot explain the full pattern of our results because reducing the symmetry of normal faces decreased attractiveness, and neither the normal- nor low-symmetry versions were blends.

Nevertheless, in Experiment 2 we attempted to rule out a blending account of our results by comparing the attractiveness of faces at three symmetry levels (normal, high, and perfect), all of which were blends (see Figure 3 and below for details). On each trial, subjects were shown two versions of the same face at different levels of symmetry and were asked to choose the more attractive one. ${ }^{6}$ Three kinds of pairs were presented for each face: the normal- and high-symmetry versions, the high- and perfect-symmetry versions, and the normal- and perfectsymmetry versions of that face. In each case, subjects should select the more symmetric version in the pair if symmetry is attractive, and given that all the images were blends, any such symmetry preference would be unlikely to result simply from a blending artifact. Alternatively, if the preference for perfectly symmetric faces in Experiment 1 was due solely to a blending artifact, we should find no preference for perfectly symmetric images in this study. In addition to choosing the more attractive face in each pair, subjects were also asked to choose the face with more appeal as a potential life partner (mate appeal) (for opposite-sex faces only).

In computer graphics, a distinction is made between two aspects of an image that can be manipulated independently, namely "shape" and "texture" information. Shape information refers to the spatial layout of landmark points or features in an image, and texture information refers to variations in the pattern of light and dark (or colors) across an image. Asymmetries can also be classified as asymmetries in shape or texture. An example of a shape asymmetry would be a difference in the position of the eyes. An example of a textural asymmetry would be a difference in brightness (or color) between corresponding regions on the two sides of a face (e.g., one pale eye and one dark eye). Blending the forward and 

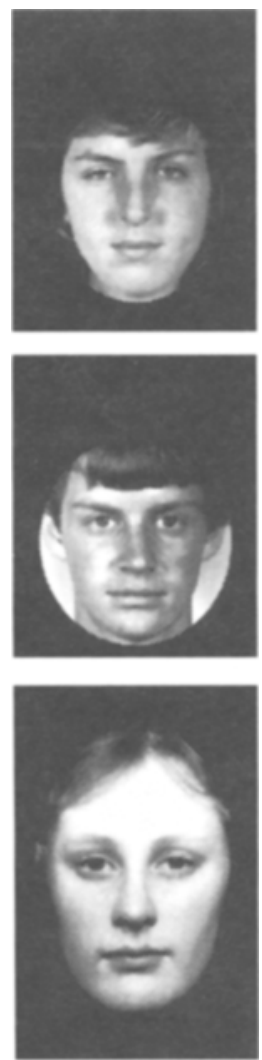

Normal
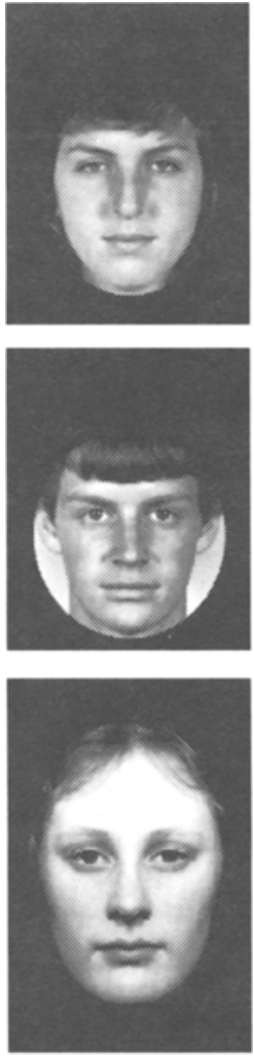

High
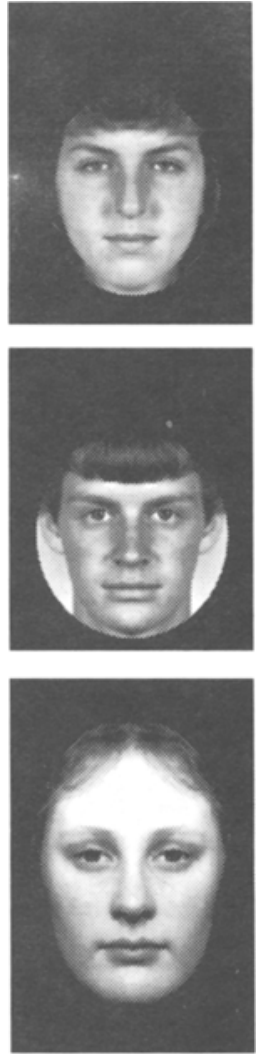

Perfect

\section{SYMMETRY}

Figure 3. Normal-, high-, and perfect-symmetry versions for three faces from Experiment 2.

mirror versions of a face results in an image with perfect bilateral symmetry of both shape and texture. The manipulation used to produce the high- and low-symmetry images in Experiment 1 (warping each face halfway toward, or away from, its perfectly symmetric configuration), however, altered only shape symmetry. Reducing shape symmetry reduced attractiveness, but increasing shape symmetry did not increase attractiveness. In Experiment 2 , we investigated whether attractiveness (and mate appeal) would increase if both texture and shape symmetry were increased in the high-symmetry versions.

Kowner (1996) has hypothesized that people are not sensitive to the subtle asymmetries present in normal faces. If she is correct, subjects should be unable to discriminate any symmetry differences between the two images in each pair. To test this claim, we also asked people to choose the more symmetric face from each pair of images.

\section{Method}

Subjects. Sixty university students ( 30 males, 30 females) received $\$ 10$ each for participating.

Stimuli. New normal versions of each face were created by blending (50:50) the low- and high-symmetry versions from Experiment I (Figure 3. left). High-symmetry versions (Figure 3. middle) were created by blending the forward and mirror images of each face in a 25:75 ratio (see Experiment 1 for general details of the blending process). If one considers a continuum of images with the normal face on the left, the mirror image on the right, and the perfectly symmetric image lying midway in between, then this $25: 75$ blend morphs the normal face halfway toward its perfectly symmetric version. In this way, we can create a high-symmetry version of each face in which both shape and textural symmetry have been increased by $50 \%$. Perfect-symmetry versions were those used in Experiment I (Figure 3, right). These images were displayed in the same oval masks as in Experiment 1.

Three pairings were created for each face, one consisting of the normaland high-symmetry versions, one consisting of the high- and perfectsymmetry versions, and the other consisting of the normal- and perfectsymmetry versions. Each of the three face pairs for a face was assigned to a different booklet, with booklets balanced for sex of face, type of pair, and left-right arrangement of faces in the pairs (less symmetric face on left or right). Additional male-only and female-only booklets were created for use in the mate appeal ratings.

Procedure. Each subject made forced choices on attractiveness, mate appeal (opposite-sex faces only), and symmetry for all the face pairs in all the booklets, with trials blocked by rating scale. Order of attractiveness and mate appeal choices was counterbalanced with order of booklets. Symmetry choices were always made last so that attention would not be drawn to symmetry before the attractiveness and mate appeal judgments were made. Note that symmetry judgments were included to determine whether subjects could accurately detect the differences in symmetry introduced by our manipulations. and not to determine whether symmetry correlated with attractiveness (or mate appeal). Therefore, we did not obtain the symmetry and attractiveness (and mate appeal) judgments from independent groups of subjects? 


\section{Results and Discussion}

The dependent variable for each rating scale was a symmetry preference score, calculated as the proportion of trials on which the more symmetric member of each pair was chosen. ${ }^{8} \mathrm{~A}$ score greater than .50 indicates a preference for the more symmetric members of the pairs. Separate three-way ANOVAs were carried out on the mean symmetry preference scores for attractiveness and symmetry choices, with sex of subject as a between-subjects factor and sex of face and pair type as repeated measures factors. The levels of pair type were normal-high, highperfect, and normal-perfect. A two-way ANOVA was carried out on the mean symmetry preference scores for mate appeal choices, with sex of subject as a betweensubjects factor and pair type as a repeated measures factor. Planned $t$ tests were carried out to test whether symmetry preferences were significantly greater than .50 . Tukey tests were used for other, unplanned comparisons.

Attractiveness. For all three pair types, there was a significant preference for the more symmetric face in the pair (i.e., mean preference scores were significantly greater than .50 ; all $t \mathrm{~s}>11.18, p \mathrm{~s}<.001$ ). Therefore, when choosing between two versions of the same face, subjects consistently preferred the more symmetric one. There was a significant main effect of pair type $[F(2,116)=$ $77.89, p<.0001$; Figure 4]. Not surprisingly, the strongest symmetry preference was for the pairs with the greatest symmetry difference, namely the normal-perfect pairs $(M=.77)$. The next highest preference was for normal-high pairs $(M=.73)$, followed by high-perfect pairs $(M=.61$, all $p$ s $<.05$, Tukey tests $)$. There was a marginal interaction between pair type and sex of subject

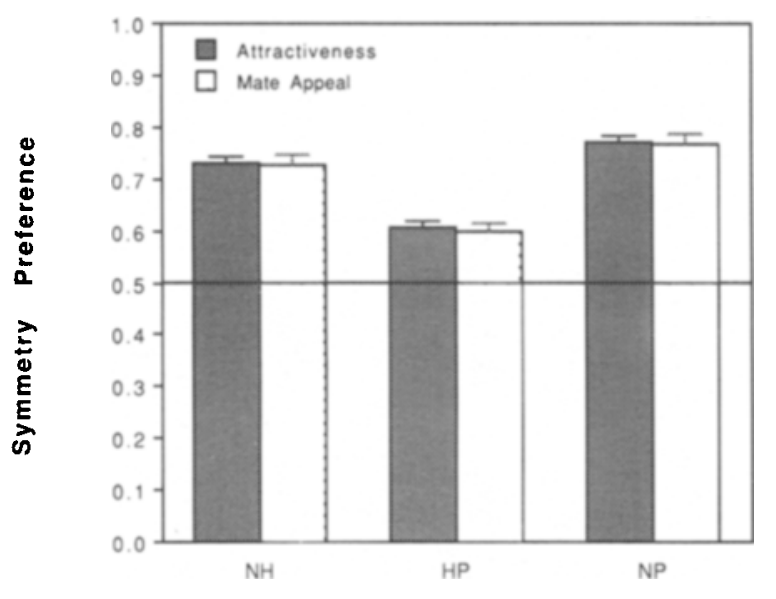

Type of Comparison

Figure 4. Mean symmetry preference as a function of type of comparison (pair type) in Experiment 2. Choice pairs were always versions of the same face. Symmetry preference values greater than 0.5 indicate a bias to select the more symmetric member of each pair. $S E$ bars are shown. NH, choices between normal- and high-symmetry versions; HP, choices between highand perfect-symmetry versions; NP, choices between normaland perfect-symmetry versions.
$[F(2,116)=2.39, p<.10]$. Males had stronger symmetry preferences than females for normal-high $(M=.76$, males; $M=.71$, females) and high-perfect ( $M=.63$, males; $M=.58$, females) pairs, but not for normal-perfect ( $M=.77$, males; $M=.77$, females) pairs. These results confirm the attractiveness of facial symmetry and demonstrate that it is not a blending artifact.

Mate appeal. The results were very similar to those obtained for attractiveness choices. For all three pair types, there was a significant preference for the more symmetric member of the pair (all $t \mathrm{~s}>9.90, p \mathrm{~s}<.001$ ). There was a significant main effect of pair type $[F(2,116)=75.60$, $p<.0001$; Figure 4], with the highest symmetry preference for normal-perfect pairs $(M=.77)$, followed by normal-high pairs $(M=.73)$, followed by high-perfect pairs $(M=.60$, all $p$ s $<.01$, Tukey tests $)$. There was also a significant main effect of sex of subject $[F(1,58)=9.84$, $p<.003$ ], with males $(M=.75)$ showing a stronger overall preference for symmetry than females $(M=.65)$.

Symmetry. In this task, a symmetry preference represents accurate performance (i.e., the most symmetric face was chosen as more symmetric). For all three pair types, performance was significantly better than chance (all $p \mathrm{~s}<.001)$. There was a significant main effect of pair type $[F(2,116)=83.03, p<.0001]$, with best performance on normal-perfect pairs $(M=.94)$, followed by normal-high pairs $(M=.88)$, followed by high-perfect pairs $(M=.81$, all $p$ s $<.01$, Tukey tests $)$. The greater sensitivity to the symmetry difference in normal-high than in high-perfect pairs may be a perceptual learning effect resulting from more experience at discriminating variations in symmetry close to normal levels. This ordering of sensitivity to symmetry differences in the three types of pairs matches, and may account for, differences in the strength of the symmetry preference for the three types of pair in the attractiveness judgments (Figure 4). The main effect of pair type was qualified by a significant interaction with sex of face $[F(1,58)=4.74, p<.02]$. The pattern of accuracy found for the three pair types (see above) did not differ for male and female faces (see above), but accuracy was higher for male than for female faces in the normal-high and normal-perfect pairs. There was a significant main effect of sex of face $[F(1,58)=51.58, p<$ $.0001]$, which was qualified by an interaction with sex of subject $[F(1,58)=4.59, p<.04]$. Symmetry was judged more accurately in male $(M=.90)$ than in female faces $(M=.85)$, and this difference was greater for male $(M=$ .91 , male faces; $M=.86$, female faces) than for female subjects ( $M=.88$, male faces; $M=.85$, female faces).

\section{GENERAL DISCUSSION}

We have shown that the attractiveness of individual faces can be increased by increasing the bilateral symmetry of those faces, that attractiveness is reduced when symmetry levels are decreased, and that perfectly symmetric faces, although not strikingly beautiful, are preferred to faces with lower levels of symmetry. Because faces are approximately bilaterally symmetric, our manipulation of symmetry did not dramatically alter the faces (see Figures 1 and 3). Nevertheless people 
were sensitive to the rather subtle differences in symmetry that resulted and preferred higher levels of symmetry.

We also found that attractiveness was associated with natural variations in symmetry between different faces (for both undistorted faces and sets of images covering an extended range of symmetry levels). These results corroborate our experimental evidence for the attractiveness of facial symmetry, described above. In addition, they replicate earlier positive correlations between symmetry and attractiveness (Grammer \& Thornhill, 1994; Jones \& Hill, 1993; Zebrowitz et al., 1996) They are also consistent with a recent report that symmetry differences between identical twins correlate positively with differences in attractiveness (Mealey \& Townsend, 1998).

Both male and female subjects found symmetry attractive in oppositesex faces, but the preference appeared to be stronger for males. This sex difference is consistent with the finding that physical appearance plays a larger role in the mate choices of males than females (Buss, 1987; Buss \& Schmitt, 1993). It is less consistent with parental investment theory (Trivers, 1972), which predicts greater female sensitivity to signals of mate quality (assuming that symmetry is a signal of mate quality) because their reproductive investment is greater than that of males. We also note that Grammer and Thornhill (1994) found very similar correlations between facial symmetry and attractiveness of oppositesex faces for males and females, and suggest that the sex difference found in our experiments be viewed with caution.

Kowner (1996) and others (Langlois et al., 1994; Samuels et al., 1994) have reported that perfect symmetry in faces is unattractive. We suggest that their results may reflect the use of perfectly symmetric chimeras, which introduce structural abnormalities (see introduction) and are therefore likely to be unattractive. Our results indicate that when these abnormalities are avoided, by blending normal and mirror images of faces, the resulting perfectly symmetric images are more attractive than the original faces. Moreover, this result did not appear to be an artifact of the attractiveness of blends per se because perfectly symmetric blends were preferred to other, less symmetric, blends.

Since we began these studies, Swaddle and Cuthill (1995) have reported that symmetry is unattractive, using a similar symmetry manipulation to ours. We suspect that their result is due to differences in facial expression that covaried with symmetry level in their study. Expression was not controlled, and the sample face shown in their paper had a small, asymmetric smile, which disappeared as symmetry increased. Smiles are attractive (see, e.g., Cunningham et al., 1995), so if this sample face is typical, then Swaddle and Cuthill's faces would have become less attractive as symmetry increased and they lost their smiles. Symmetric smiles may also be unnatural (Kowner, 1996), which could restrict the appeal of perfect symmetry for smiling faces. We are currently investigating the effects of expression on the attractiveness of symmetry. Another feature of Swaddle and Cuthill's stimuli that could have minimized the appeal of symmetry is that only the internal features of faces were shown, thereby eliminating cues to mate quality provided by the jaw and chin. Development of this part of the face is strongly influenced by sex hormones, which stress the immune system (Thornhill \& Gangestad, 1996), and symmetry in that region may therefore provide a powerful cue to mate quality. Our results show that when emotional expression is carefully controlled (and neutral), and the whole face is visible, symmetry is attractive. Similar results have recently been obtained by Perrett and his colleagues (Perrett, Burt, Lee, Rowland, \& Edwards, 1998).

The hypothesis that facial symmetry is attractive was derived from evolutionary theory. We therefore collected mate appeal ratings, as wel as attractiveness ratings, to gain preliminary information about whether symmetry might influence human mate choice. The mate appeal results were similar to those described above for attractiveness, with more symmetric images being rated as more appealing as a potential life partner than less symmetric images. The sex difference found for attractiveness was even greater for mate appeal ratings, with only males showing replicable correlations between symmetry and mate appeal. More direct measures of the impact of facial symmetry on reproductive behavior will be needed to determine whether facial symmetry (like bodily symmetry. Thornhill \& Gangestad. 1994) influences mate choice, but the present results suggest that it may do so.
In the introduction, we noted that the presence of directional asymmetries in faces means that some degree of asymmetry is normal, and that not all facial asymmetries would indicate a poor-quality mate. It was not, therefore, obvious that perfect facial symmetry would be attractive (or even that it should be considered optimal in a system tuned to detect fluctuating asymmetries). Does our finding that people prefer perfectly symmetric faces to less symmetric versions mean that perfect symmetry is attractive despite its abnormality? We suspect not, because directional asymmetries in resting faces appear to be very small, which means that perfect symmetry is not abnormal in the sense of deviating markedly from the population mean. Rhodes, Sumich, and Byatt (in press) found that the average female face (made by averaging female faces together to eliminate fluctuating, but not directional, asymmetries) and its perfectly symmetric counterpart were perceived as equally symmetric. The male average was considered less symmetric than its perfectly symmetric counterpart, but still appeared more symmetric than any individual male face. Therefore, directional asymmetries in faces (especially female faces) appear to be very small, so most facial asymmetries would be fluctuating asymmetries, which could potentially signal mate quality. It is an open question whether the slight degree of asymmetry present in the average male face (due to directional asymmetries) would be more attractive than perfect symmetry. Future studies using a finer grained manipulation of symmetry levels than that used in the present experiments would be needed to answer this question.

If facial symmetry is a standard of beauty set by nature rather than culture, then how might a preference for symmetry have evolved? In the introduction, we raised the possibility that facial symmetry, like symmetry in other morphological traits (for an extensive review, see Thornhill \& Møller, 1997), may be a reliable signal of health and genetic quality. If it is, individuals who prefer to mate with symmetric individuals would have higher fitness, on average, than those without a preference for symmetric mates, and the symmetry preference would be selected for. This possibility receives preliminary support from Shackelford and Larsen's (1997) results, described above.

There are, however, other ways that a symmetry preference could evolve. Given that symmetry is heritable (Møller \& Thornhill, 1997), the offspring of individuals who chose symmetric mates would tend to be symmetric and therefore popular as mates (as long as the preference for symmetry was also heritable). This sort of feed-forward mechanism can maintain preferences in a population (Fisher, 1915, 1930). A preference for facial symmetry could also be a by-product of some general sensitivity to symmetric patterns that has evolved for reasons that have nothing to do with assessing mate quality. ${ }^{9}$ For example, sensitivity to symmetry could have evolved because it is useful in form perception generally. Support for this "perceptual bias" hypothesis comes from simulation studies showing that symmetry preferences evolve when connectionist networks are trained to recognize patterns (Enquist \& Arak, 1994; Johnstone, 1994) and from evidence that symmetric patterns generally are attractive (Corballis \& Beale, 1976). Note that neither the feed-forward mechanism nor the perceptual bias account requires that symmetry signals mate quality or that a symmetry preference is specific for faces (or bodies) for such a preference to evolve.

Our results are consistent with the notion that symmetry is a standard of beauty set by nature, but they do not allow us to rule out an alternative, culturally based account. Cross-cultural and developmental studies are potentially informative. Evidence of cross-cultural agreement on the attractiveness of facial symmetry would support the biological view, as would evidence of early emergence of the preference (so that there is little opportunity for cultural shaping) or emergence at puberty (triggered by the sex hormones that motivate the search for a mate). Additional studies are also needed to replicate and extend Shackelford and Larsen's (1997) initial evidence for an association between facial symmetry and health.

Several commentators have suggested that a preference for symmetry may underlie our preference for averageness because average faces are more symmetric than other faces (see Langlois et al., 1994, for a review). Our finding that symmetry is attractive adds plausibility to this conjeeture, which was advanced in the absence of good evidence that symmetry is indeed attractive. Nevertheless. we think it unlikely that a preference for symmetry will completely account for the attractiveness of average- 
ness. Langlois and her colleagues (1994) have shown that a blend of a large number of different faces is more attractive than perfectly symmetric versions of individual faces like those used here. This result suggests that an average facial configuration, obtained by blending many different faces, is more attractive than perfect symmetry. We are currently investigating the precise relationship between preferences for symmetry and averageness. Our results suggest that symmetry and averageness make independent contributions to attractiveness (Rhodes et al., in press).

In the last decade, scholars from a variety of disciplines have become interested in the evolution of the mind (e.g., Barkow, Cosmides, \& Tooby, 1992; Corballis, 1991; Dennett, 1995; Pinker, 1994, 1997). A clear consensus has emerged that evolutionary forces have shaped our perceptual, communicative, and reasoning systems. The perception of facial attractiveness seems especially likely to be under selection pressure, given the importance of facial attractiveness in human mate choice (Buss \& Schmitt, 1993). Our results lend preliminary support to this view.

\section{REFERENCES}

Barkow, J. H., Cosmides, L., \& ToOBY, J. (1992). The adapted mind: Evolutionary psychology and the generation of culture. New York: Oxford University Press.

BEALE, J. M., \& KeIL, F. C. (1995). Categorical effects in the perception of faces. Cognition, 57, 217-239

Benson, P., \& Perrett, D. (1992, February 22). Face to face with the perfect image. New Scientist, No. 1809, 32-35.

BOROD, J. C. (1993). Cerebral mechanisms underlying facial, prosodic, and lexical emotional expression: A review of neuropsychological studies and methodological issues. Neuropsychology, 7, 445-463.

Brooks, M., \& PomianKowski, A. (1994). Symmetry is in the eye of the beholder. Trends in Ecology \& Evolution, 9, 201-202.

Buss, D. M. (1987). Sex differences in human mate selection criteria An evolutionary perspective. In C. Crawford, M. Smith, \& D. Krebs (Eds.), Sociobiology and psychology: Ideas, issues and applications (pp. 335-351). Hillsdale, NJ: Erlbaum.

Buss, D. M., \& Schmitt, D. P. (1993). Sexual strategies theory: An evolutionary perspective on human mating. Psychological Review, 100, 204-232.

CoNCAR, D. (1995). Sex and the symmetrical body. New Scientist, 146, 40-44.

Corballis, M. C. (1991). The lop-sided ape. New York: Oxford University Press.

Corballis, M. C., \& Beale, I. L. (1976). The psychology of left and right. Hillsdale, $\mathrm{NJ}$ : Erlbaum.

CRONIN, H. (1991). The ant and the peacock: Altruism and sexual selection from Darwin to today. Cambridge: Cambridge University Press.

Cunningham, M. R., Roberts, A. R., Wu, C.-H., Barbee, A. P., \& DRUEN, P. B. (I995). "Their ideas of beauty are, on the whole, the same as ours": Consistency and variability in the cross-cultural perception of female physical attractiveness. Journal of Personality \& Social Psychology, 68, 261-279.

DENNETT, D. C. (1995). Darwin's dangerous idea: Evolution and the meanings of life. New York: Simon \& Shuster.

ENQUIST, M., \& ARAK, A. (1994). Symmetry, beauty and evolution. $\mathrm{Na}$ ture, 372, 169-172.

FISHER, R. A. (1915). The evolution of sexual preference. Eugenics Review, 7, 184-192.

FISHER, R. A. (1930). The genetical theory of natural selection. Oxford: Clarendon Press.

Gangestad, S. W., \& Buss, D. M. (1993). Pathogen prevalence and human mate preferences. Ethology \& Sociobiology, 14, 89-96.

Grammer, K., \& ThoRNhill, R. (1994). Human (Homo sapiens) facial attractiveness and sexual selection: The role of symmetry and averageness. Journal of Comparative Psychology, 108, 233-242.

JOHNSTONE, R. A. (1994). Female preference for symmetrical males as a by-product of selection for mate recognition. Nature, 372, 172-175

JoNES, D., \& HILL, K. (1993). Criteria of facial attractiveness in five populations. Human Nature, 4, $271-296$.

KOWNER, R. (1996). Facial asymmetry and attractiveness judgment in developmental perspective. Journal of Experimental Psychology: Human Perception \& Performance, 22, 662-675.

LANGLOIS, J. H., \& RogGman, L. A. (1990). Attractive faces are only average. Psychological Science, 1, 115-121.

Langlois, J. H., Roggman, L. A., Casey, R. J., Ritter, J. M., RieserDANNER, L. A., \& JENKInS, V. Y. (1987). Infant preferences for attractive faces: Rudiments of a stereotype? Developmental Psychology, 23, 363-369.

Langlois, J. H., Roggman, L. A., \& Musselman, L. (1994). What is average and what is not average about attractive faces? Psychological Science, 5, 214-220.

Mealey, L., \& Townsend, G. C. (1998). The role of fluctuating asymmetry on judgments of physical attractiveness: A monozygotic cotwin comparison. In J. Kieser \& L. Mealey (Eds.). Dento-facial variation in perspective. Singapore: World Scientific Publishing.

Møller, A. P., \& PomianKowski, A. (1993). Fluctuating asymmetry and sexual selection. Genetica, 89, 267-279.

Møller, A. P., \& Thornhill, R, (1997). A meta-analysis of the heritability of developmental stability. Journal of Evolutionary Biology, 10, 1-16.

Palmer, A. R., \& Strobeck, C. A. (1986). Fluctuating asymmetry: Measurement, analysis, pattern. Annual Review of Ecology \& Systematics, 17, 391-421.

Parsons, P. A. (1990). Fluctuating asymmetry: An epigenetic measure of stress. Biological Review, 65, 131-145.

Perrett, D. I., Burt, D. M., Lee, K. J., Rowland, D. A., \& Edwards, R. E. (1998). Fluctuating asymmetry in human faces: Symmetry is beautiful. Unpublished manuscript.

Perrett, D. I., May, K. A., \& Yoshikawa, S. (1994). Facial shape and judgements of female attractiveness. Nature, 368, 239-242.

PINKER, S. (1994). The language instinct: The new science of language and mind. London: Penguin.

PINKER, S. (1997). How the mind works. New York: Norton.

Previc, F. H. (1991). A general theory concerning the prenatal origins of cerebral lateralization in humans. Psychological Review, 98, 299-334.

RHODEs, G. (1996). Superportraits: Caricatures and recognition. Hove, U.K.: Psychology Press.

Rhodes, G., Sumich, A., \& Byatt, G. (in press). Are average facial configurations only attractive because of their symmetry? Psychological Science.

Rhodes, G., \& Tremewan, T. (1996). Averageness, exaggeration, and facial attractiveness. Psychological Science, 7, 105-110.

RIDLEY, M. (1992). Swallows and scorpionflies find symmetry is beautiful. Science, 257, 327-328.

Samuels, C. A., Butterworth, G., Roberts, T., Graupner, L., \& HoLE, G. (1994). Facial aesthetics: Babies prefer attractiveness to symmetry. Perception, 23, 823-831.

Shackelford, T. K., \& LaRSEN, R. J. (1997). Facial asymmetry as an indicator of psychological, emotional, and physiological distress. Journal of Personality \& Social Psychology, 72, 456-466.

SwadDLE, J. P., \& CuTHILl, I. C. (1995). Asymmetry and human facial attractiveness: Symmetry may not always be beautiful. Proceedings of the Royal Society of London: Series B, 261, 111-116.

Symons, D. (1979). The evolution of human sexuality. Oxford: Oxford University Press.

Thornhill, R., \& GANGestad, S. W. (1993). Human facial beauty. Human Nature, 4, 237-269.

THORNHILL, R., \& GANGESTAD, S. W. (1994). Human fluctuating asymmetry and sexual behavior. Psychological Science, 5, 297-302.

THORNHILL, R., \& GANGESTAD, S. W. (1996). The evolution of human sexuality. Trends in Ecology \& Evolution, 11, 98-102.

ThORNhILl, R., \& Møller, A. P. (1997). Developmental stability, disease and medicine. Biological Reviews, 72, 497-548.

Trivers, R. L. (1972). Parental investment and sexual selection. In B. Campbell (Ed.), Sexual selection and the descent of man: $1871-$ 197I (pp. 136-179). Chicago: Aldine.

Watson, P. M., \& THORNhilL, R. (1994). Fluctuating asymmetry and sexual selection. Trends in Ecology \& Evolution, 9, 21-25.

Zebrowitz, L. A., Volnescu, L., \& Collins, M. A. (1996). Wide- 
eyed and crooked-faced-Determinants of perceived and real honesty across the lifespan. Personality \& Social Psychology Bulletin, 22, 1258-1269.

\section{NOTES}

1. Here and elsewhere, the claim that a particular characteristic is at tractive should be interpreted in the relative sense of attractiveness varying with the level of that characteristic, rather than in an absolute sense.

2. Although average faces are attractive, they may not be the most attractive faces. For example, the most attractive female faces appear to differ systematically from average in some respects, having relatively large eyes, high cheekbones, small jaws and chins, and short nose-tomouth distances (Perrett et al., 1994). Exaggerating these deviations from averageness increases attractiveness further. For some traits, then extreme values may be preferred to average values (see Cronin, 1991, and Rhodes, 1996, for discussions of the possible adaptive value of preferences for certain extreme traits).

3. This preference for asymmetric faces was found for the faces of children and young adults, but not for elderly adults. Symmetric versions were preferred for elderly faces, apparently because they looked younger.

4. A follow-up analysis of opposite-sex attractiveness ratings showed the same interaction $[F(3,186)=4.54, p<.005]$, with the same pattern of means, although the sex difference for perfectly symmetric images (or at any other symmetry level) was not significant by a simple test of main effects.
5. Note that the attractiveness and mate appeal ratings could not have been influenced by making symmetry ratings because symmetry was always rated last, so the results of the ANOVAs on those variables cannot have been affected by making symmetry ratings.

6 . This forced-choice procedure follows that used in other studies investigating facial attractiveness. For example, Kowner (1996) required subjects to make forced choices between natural and perfectly symmetric versions of faces, and Perrett et al. (1994) asked subjects to make forced choices between even more similar pairs of images (composites that differed in the number and selection of component images).

7. It is possible that making attractiveness and mate appeal judgments for opposite- (but not same-) sex faces contributes to any similarities found for these two tasks. However, comparison of the correlations between attractiveness and mate appeal in Experiments 1 (same raters) and $1 \mathrm{~A}$ (independent raters) suggests that the contribution of any such carryover effects would be quite small.

8. Note that separate proportions (symmetry scores) were calculated for each subject for each cell of the design, so that an ANOVA on such proportions is quite proper.

9. Note that more than one kind of selection pressure can operate on a given trait or preference (see Rhodes, 1996, for further discussion).

(Manuscript received October 10, 1996; revision accepted for publication January 14, 1998.) 